\title{
Long-term Effects of Intravenous Cyclophosphamide in Combination with Mesna Provided Intravenously and via Bladder Perfusion in a Patient with Severe Multifocal Motor Neuropathy
}

\author{
Eri Iwasawa ${ }^{1}$, Takuya Ohkubo ${ }^{1}$, Tadashi Kanouchi ${ }^{2}$, Takashi Kanda ${ }^{3}$, \\ Hidehiro Mizusawa ${ }^{4}$ and Takanori Yokota ${ }^{1}$
}

\begin{abstract}
A 25-year-old woman presenting with progressive muscle weakness in the distal extremities in the absence of sensory involvement for 2 years was diagnosed with multifocal motor neuropathy (MMN). Her disease was difficult to manage with various immunosuppressants, and the muscle weakness eventually progressed to involve the respiratory muscles, necessitating mechanical ventilation. Intravenous cyclophosphamide (CY) dramatically improved her symptoms, and she has since maintained her ambulatory status for 18 years with intermittent CY therapy. Because the patient presented with hemorrhagic cystitis due to CY, we also implemented mesna administration by bladder perfusion. The administration of CY should therefore be considered in patients with severe MMN that is unresponsive to standard therapy.
\end{abstract}

Key words: multifocal motor neuropathy (MMN), cyclophosphamide

(Intern Med 56: 1893-1896, 2017)

(DOI: 10.2169/internalmedicine.56.8157)

\section{Introduction}

Multifocal motor neuropathy (MMN) is an acquired autoimmune demyelinating neuropathy that manifests in patients as asymmetrical limb weakness without sensory involvement. MMN is diagnosed based on the clinical features and electrodiagnostic criteria from nerve conduction studies. In addition, the presence of immunoglobulin $\mathrm{M}$ antiganglioside GM1 antibody (anti-GM1 antibody) in serum is supportive of a diagnosis of MMN. Although many MMN cases present with relatively mild weakness, there are some cases that involve the respiratory muscles $(1,2)$ or present with severe weaknesses of the extremities. Accordingly, prompt and precise treatment is critical for the management of MMN.

Intravenous immunoglobulin (IVIg) is the first-line treatment for MMN. Although cyclophosphamide (CY) has been reported to be effective in several cases (3-6), it is less frequently recommended due to potential toxicities, including myelosuppression, hemorrhagic cystitis, and possible carcinogenicity (7). We herein report a case of MMN in which the patient regained ambulatory status with the assistance of a cane from a bedridden and respirator-dependent state after intermittent intravenous CY (IVCY) treatment combined with mesna (sodium 2-mercaptoethane sulfonate; Uromitexan ${ }^{\circledR}$ ) delivered intravenously and through bladder perfusion. This improvement has been maintained for 18 years with intermittent $\mathrm{CY}$ treatment. The present case provides an important contribution to the current knowledge about MMN treatment.

\section{Case Report}

A 25-year-old woman presented with chronic progressive muscle weakness in her distal extremities in the absence of

\footnotetext{
${ }^{1}$ Department of Neurology and Neurological Science, Graduate School of Medicine, Tokyo Medical and Dental University, Japan, ${ }^{2}$ Clinical Laboratory, Medical Hospital of Tokyo Medical and Dental University, Japan, ${ }^{3}$ Department of Neurology and Clinical Neuroscience, Yamaguchi University Graduate School of Medicine, Japan and ${ }^{4}$ National Institute of Neuroscience, National Center of Neurology and Psychiatry, Japan Received for publication August 22, 2016; Accepted for publication October 30, 2016 Correspondence to Dr. Takanori Yokota, tak-yokota.nuro@tmd.ac.jp
} 
any sensory symptoms for 2 years. The weakness was initially asymmetrical and more evident in the upper limbs than in the lower extremities. Her intrinsic muscles of the hands were atrophied, with a decreased tendon reflex in the upper and lower extremities. The findings on a sensory examination were normal. The results of a motor nerve conduction study showed moderate slowing $(35.7 \mathrm{~m} / \mathrm{s}$ for the median nerve) and focal conduction blocks in the right median, ulnar, tibial, and peroneal nerves (7), based on the electrodiagnostic criteria of the European Federation of Neurological Societies/Peripheral Nerve Society Guideline (8). The results of sensory nerve conduction studies were completely normal. In addition, IgM anti-GM1 antibody titer was highly elevated to $1: 6,400$ (normal range $<1: 100$ ) in the patient's serum. She was accordingly diagnosed with MMN. One year prior to the onset of muscle weakness, she was also diagnosed with systemic lupus erythematosus (SLE), meeting 4 of the 11 American College of Rheumatology criteria (photosensitivity, malar rash, positive antinuclear antibody, and lupus anticoagulant). The patient soon became negative for autoantibodies, and her SLE did not become active again after 28 years of age.

For the treatment of MMN, oral prednisolone (15 mg/ day), plasma exchange, and intravenous methylprednisolone (1 g/day) for 3 days had little effect. Subsequent treatment with IVIg $(2 \mathrm{~g} / \mathrm{kg})$ over a period of 5 days provided a slight improvement in symptoms, however, it later quickly relapsed. Finally, an oral regimen of CY (100 mg/day) improved the patient's symptoms and allowed her to remain stable for 1 year. When the patient was 32 years old, she experienced severe hemorrhagic cystitis; her hemoglobin level decreased from $10.7 \mathrm{~g} / \mathrm{dL}$ to $5.5 \mathrm{~g} / \mathrm{dL}$ which thus necessitated a massive transfusion of red blood cell concentrates. CY was reluctantly discontinued. Treatment with azathioprine $(150 \mathrm{mg} /$ day) led to pancytopenia, and her limb weakness progressed despite a regimen of cyclosporine A (450 $\mathrm{mg} /$ day).

After the discontinuation of CY, the patient's weakness progressed gradually. She became unable to walk and needed to use a wheelchair. After 5 doses of interferon alpha-2a (3 million units, intramuscularly), her symptoms worsened, and her muscle weakness progressed to include the respiratory and swallowing muscles. Because of dysphagia and hypercapnia due to a decreased vital capacity, the patient was intubated and required mechanical ventilation. Two courses of IVIg $(2 \mathrm{~g} / \mathrm{kg})$ over a period of 5 days did not offer any improvements, so IVCY (600 mg; $430 \mathrm{mg}$ / $\mathrm{m}^{2}$ ) was started in combination with mesna administered both intravenously $(1,200 \mathrm{mg}$, three times per day) and by bladder perfusion (400 mg in 1,000 mL normal saline 3-8 times per day for 5 days) to protect the bladder from further episodes of hemorrhagic cystitis. The patient began to recover and was eventually weaned from mechanical ventilation. After 3 courses of IVCY (600 mg), her manual muscle test scores improved from 0 to 2 in all extremities. Subsequent IVCY (800 mg; $570 \mathrm{mg} / \mathrm{m}^{2}$ ) at an interval of 2-to-4 weeks enabled her to walk again and improved the amplitudes and velocities of the compound muscle action potentials (CMAPs) in nerve conduction studies.

We maintained the patient's ambulatory status by continuing intermittent IVCY therapy, since IVIg alone seemed to be insufficient once she began to experience weakness. Intermittent IVCY with mesna administered intravenously and through bladder perfusion was continued for 18 years; during this time, we gradually lengthened the interval between IVCY infusions. With a treatment interval of 12 weeks, the patient had no muscle weakness, and nerve conduction studies also revealed improvement. After 18 years of continued treatment, we attempted to extend the interval to 36 weeks, but the patient's disease strikingly relapsed with severe weakness and decreased CMAP amplitudes and velocities (Figure). We are now trying to manage these relapses with a combination of more frequent IVCY, IVIg, and mycophenolate mofetil therapy.

The patient has not experienced hemorrhagic cystitis since the last episode reported at 32 years of age, and no malignancies have been identified in yearly cancer screenings with blood tests, a urinalysis, computed tomography, and gastrointestinal fiberscopy.

\section{Discussion}

This case report is an important demonstration of the utility of CY therapy to prevent MMN relapse for a period of 18 years. In the present case, IVIg as first-line therapy was insufficient to treat the symptom exacerbations and improve her muscle weakness; in contrast, CY therapy led to substantial symptom improvements, and its intermittent use not only helped the patient maintain her muscle strength but also improved her nerve conduction study results. Of note, this patient had several characteristics atypical of MMN. First, there have been few reported cases of MMN involving the respiratory muscles $(1,2)$. Second, although the limb weakness was initially asymmetrical in this case, it later became relatively symmetrical and predominantly involved the distal muscles; this made the diagnosis ambiguous between MMN and pure motor-type chronic inflammatory demyelinating polyradiculoneuropathy (9). However, the observation of an elevated serum IgM anti-GM1 antibody titer supported a diagnosis of MMN. Third, the muscle weakness in this case was remarkably exacerbated after interferon alpha administration. This might be attributable to comorbid SLE, since type 1 interferon (especially interferon alpha) is thought to contribute to its pathogenesis (10).

The pathogenesis of MMN is not completely understood, but the presence of serum autoantibodies against ganglioside and its possible effect on complement deposition at the nodes of Ranvier (11) suggest that the mechanism involves humoral immunity. CY depletes lymphocytes, including B lymphocytes (12), in order to exert its immunosuppressive effect and therefore may target the dysregulation of autoimmunity in MMN. Currently, a limited number of stud- 


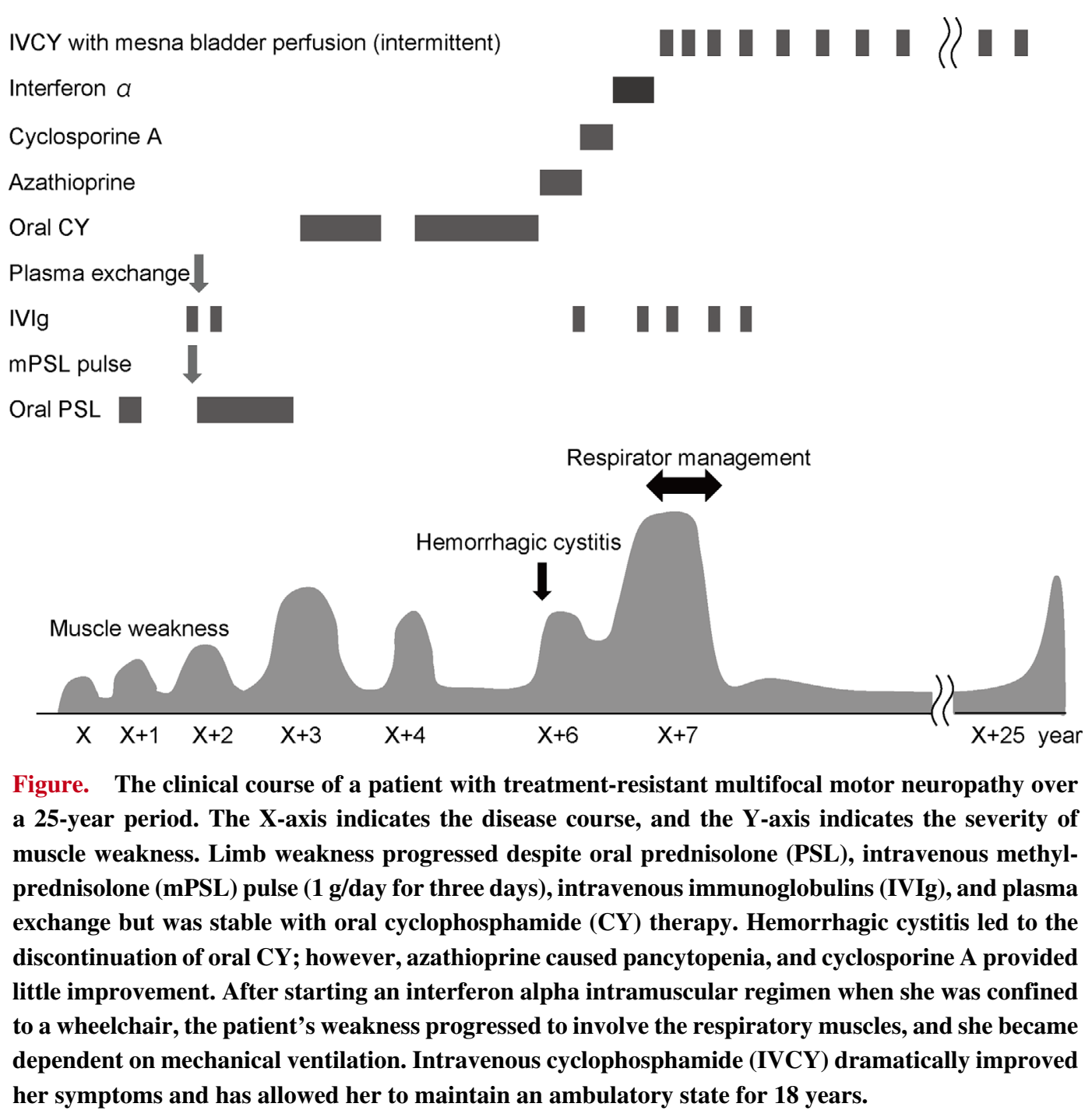

ies (3-6) support the use of CY for the treatment of MMN, particularly considering the potential toxicities of this therapy (6). However, in contrast to immunosuppressants such as rituximab and eculizumab, which have been proposed as a therapeutic regimen for MMN but failed to show their effectiveness independent of IVIg $(13,14)$, CY was markedly effective in our case. Of particular note was its ability to maintain MMN remission for as long as 18 years. Some cases of MMN have reportedly shown insufficient responses to IVIg from treatment onset, or the efficacy of IVIg was gradually diminished after several years $(15,16)$. We therefore propose $\mathrm{CY}$ as a second-line therapeutic for such patients.

We presented a new protocol for the administration of $\mathrm{CY}$ in combination with mesna intravenously and through bladder perfusion as a way of reducing the risk of hemorrhagic cystitis. Mesna is exclusively excreted in urine after intravenous administration and exerts its detoxification effect by first directly binding to and inactivating urotoxic acrolein, which is a metabolite of cyclophosphamide, and also by stabilizing 4-hydroxy-cyclophosphamide (a primary metabolite of cyclophosphamide that is metabolized into acrolein unless interrupted) and preventing acrolein production. We there- fore decided to start this novel intravesical administration of mesna based on its pharmacokinetics (17), which are observed primarily in the urine.

In patients with MMN that shows limited responsiveness to IVIg and is severe enough to involve the respiratory muscles, we believe that $\mathrm{CY}$ in combination with mesna should be considered, although regular cancer screening should also be implemented.

The authors state that they have no Conflict of Interest (COI).

\section{References}

1. Beydoun SR, Copeland D. Bilateral phrenic neuropathy as a presenting feature of multifocal motor neuropathy with conduction block. Muscle Nerve 23: 556-559, 2000.

2. Boonyapisit K, Katirji B. Multifocal motor neuropathy presenting with respiratory failure. Muscle Nerve 23: 1887-1890, 2000.

3. Feldman EL, Bromberg MB, Albers JW, Pestronk A. Immunosuppressive treatment in multifocal motor neuropathy. Ann Neurol 30: 397-401, 1991.

4. Meucci N, Cappellari A, Barbieri S, Scarlato G, Nobile-Orazio E. Long term effect of intravenous immunoglobulins and oral cyclophosphamide in multifocal motor neuropathy. J Neurol Neurosurg 
Psychiatry 63: 765-769, 1997.

5. Krarup C, Stewart JD, Sumner AJ, Pestronk A, Lipton SA. A syndrome of asymmetric limb weakness with motor conduction block. Neurology 40: 118-127, 1990.

6. Brannagan TH 3rd, Alaedini A, Gladstone DE. High-dose cyclophosphamide without stem cell rescue for refractory multifocal motor neuropathy. Muscle Nerve 34: 246-250, 2006.

7. Yokota T, Saito Y, Yuki N, Tanaka H. Persistent increased threshold of electrical stimulation selective to motor nerve in multifocal motor neuropathy. Muscle Nerve 19: 823-828, 1996.

8. Van Schaik IN, Leger JM, Nobile-Orazio E, et al. European Federation of Neurological Societies/Peripheral Nerve Society Guideline on management of multifocal motor neuropathy. Report of a joint task force of the European Federation of Neurological Societies and the Peripheral Nerve Society-first revision. J Peripher Nerv Syst 15: 295-301, 2010.

9. Van den Bergh PY, Hadden RD, Bouche P, et al. European Federation of Neurological Societies/Peripheral Nerve Society Guideline on management of chronic inflammatory demyelinating polyradiculopathy: report of a joint task force of the European Federation of Neurological Societies and the Peripheral Nerve Society-First Revision. Eur J Neurol 17: 356-363, 2010.

10. Elkon KB, Stone VV. Type I interferon and systemic lupus erythematosus. J Interferon Cytokine Res 11: 803-812, 2011.

11. Suzuki K, Rasband MN, Tohyama K, et al. Anti-GM1 antibodies cause complement-mediated disruption of sodium channel clusters in peripheral motor nerve fibers. J Neurosci 27: 3956-3967, 2007.

12. Hurd ER, Giuliano VJ. The effect of cyclophosphamide on B and T lymphocytes in patients with connective tissue diseases. Arthritis Rheum 18: 67-75, 1975.

13. Chaudhry V, Cornblath DR. An open-label trial of rituximab (Ri$\left.\operatorname{tuxan}^{\circledR}\right)$ in multifocal motor neuropathy. J Peripher Nerv Syst 15: 196-201, 2010.

14. Fitzpatrick AM, Mann CA, Barry S, Brennan K, Overell JR, Willison HJ. An open label clinical trial of complement inhibition in multifocal motor neuropathy. J Peripher Nerv Syst 16: 84-91, 2011.

15. Van den Berg-Vos RM, Franssen H, Wokke JHJ, Van den Berg LH. Multifocal motor neuropathy: long-term clinical and electrophysiological assessment of intravenous immunoglobulin maintenance treatment. Brain 125: 1875-1886, 2002.

16. Terenghi F, Cappellari A, Bersano A, Carpo M, Barbieri S, Nobile-Orazio E. How long is IVIg effective in multifocal motor neuropathy? Neurology 62: 666-668, 2004.

17. Brock N, Pohl J. The development of mesna for regional detoxification. Cancer Treat Rev 10: 33-43, 1983.

The Internal Medicine is an Open Access article distributed under the Creative Commons Attribution-NonCommercial-NoDerivatives 4.0 International License. To view the details of this license, please visit (https://creativecommons.org/licenses/ by-nc-nd/4.0/).

(C) 2017 The Japanese Society of Internal Medicine http://www.naika.or.jp/imonline/index.html 